\title{
The evolving landscape of sex-based differences in lung cancer: a distinct disease in women
}

\author{
Meera Ragavan ${ }^{1}$ and Manali I. Patel ${ }^{2,3,4}$ \\ Number 6 in the Series "Sex and gender in lung disease" \\ Edited by Jason Weatherald, Marc Humbert and Renata Riha
}

${ }^{1}$ Division of Hematology/Oncology, Dept of Medicine, University of California San Francisco, San Francisco, CA, USA. ${ }^{2}$ Division of Oncology, Dept of Medicine, Stanford University School of Medicine, Stanford, CA, USA. ${ }^{3}$ Division of Oncology, VA Palo Alto Healthcare System, Palo Alto, CA, USA. ${ }^{4}$ Dept of Medicine, Center for Health Policy/Primary Care Outcomes Research, Stanford University School of Medicine, Stanford, CA, USA.

Corresponding author: Meera Ragavan (meera.ragavan@ucsf.edu)

Shareable abstract (@ERSpublications)

Sex-based differences in lung cancer span the care continuum. This suggests lung cancer may increasingly be viewed as a distinct disease in women, with implications for screening and treatment. Lung cancer research should capture these sex-based differences. https://bit.ly/2WfhaB4

Cite this article as: Ragavan M, Patel MI. The evolving landscape of sex-based differences in lung cancer: a distinct disease in women. Eur Respir Rev 2022; 31: 210100 [DOI: 10.1183/16000617.0100-2021].

Abstract

Copyright @The authors 2022

This version is distributed under the terms of the Creative Commons Attribution NonCommercial Licence 4.0. For commercial reproduction rights and permissions contact permissions@ersnet.org

Received: 16 April 2021 Accepted: 16 Aug 2021

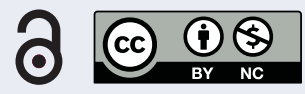

In stark contrast to a few decades ago when lung cancer was predominantly a disease of men who smoke, incidence rates of lung cancer in women are now comparable to or higher than those in men and are rising alarmingly in many parts of the world. Women face a unique set of risk factors for lung cancer compared to men. These include exogenous exposures including radon, prior radiation, and fumes from indoor cooking materials such as coal, in addition to endogenous exposures such as oestrogen and distinct genetic polymorphisms. Current screening guidelines only address tobacco use and likely underrepresent lung cancer risk in women. Women were also not well represented in some of the landmark prospective studies that led to the development of current screening guidelines. Women diagnosed with lung cancer have a clear mortality benefit compared to men even when other clinical and demographic characteristics are accounted for. However, there may be sex-based differences in outcomes and side effects of systemic therapy, particularly with chemotherapy and immunotherapy. Ongoing research is needed to better investigate these differences to address the rapidly changing demographics of lung cancer worldwide.

\section{Introduction}

The demographic makeup of lung cancer has dramatically shifted over the last few decades. Compared to men, women are more likely to be diagnosed at a younger age and with adenocarcinoma, have a family history of cancer, and lack a smoking history $[1,2]$. The pathogenesis of lung cancer in the never-smoker population is not currently well understood. A number of potential exogenous and endogenous risk factors have been proposed. Some risk factors are endogenous and may vary according to biological sex, such as differences in genetic polymorphisms. Other risk factors differ based on gender, such as differences in exogenous exposures including indoor cooking fumes, secondhand smoke and hormone replacement therapy (HRT) [3-5]. Current screening guidelines only capture smoking history and therefore may underestimate risk in women, particularly in underrepresented minorities [3, 6, 7]. While overall lung cancer outcomes are more favourable in women compared to men even when adjusted for age, smoking status and stage, the global rise in incidence of lung cancer in women is alarming and threatens to upend these patterns in the coming decades [8]. This narrative review will examine the evidence regarding sex-based differences in the epidemiology, pathogenesis and outcomes in women compared to men diagnosed with lung cancer. A summary of these differences is displayed in figure 1 . 


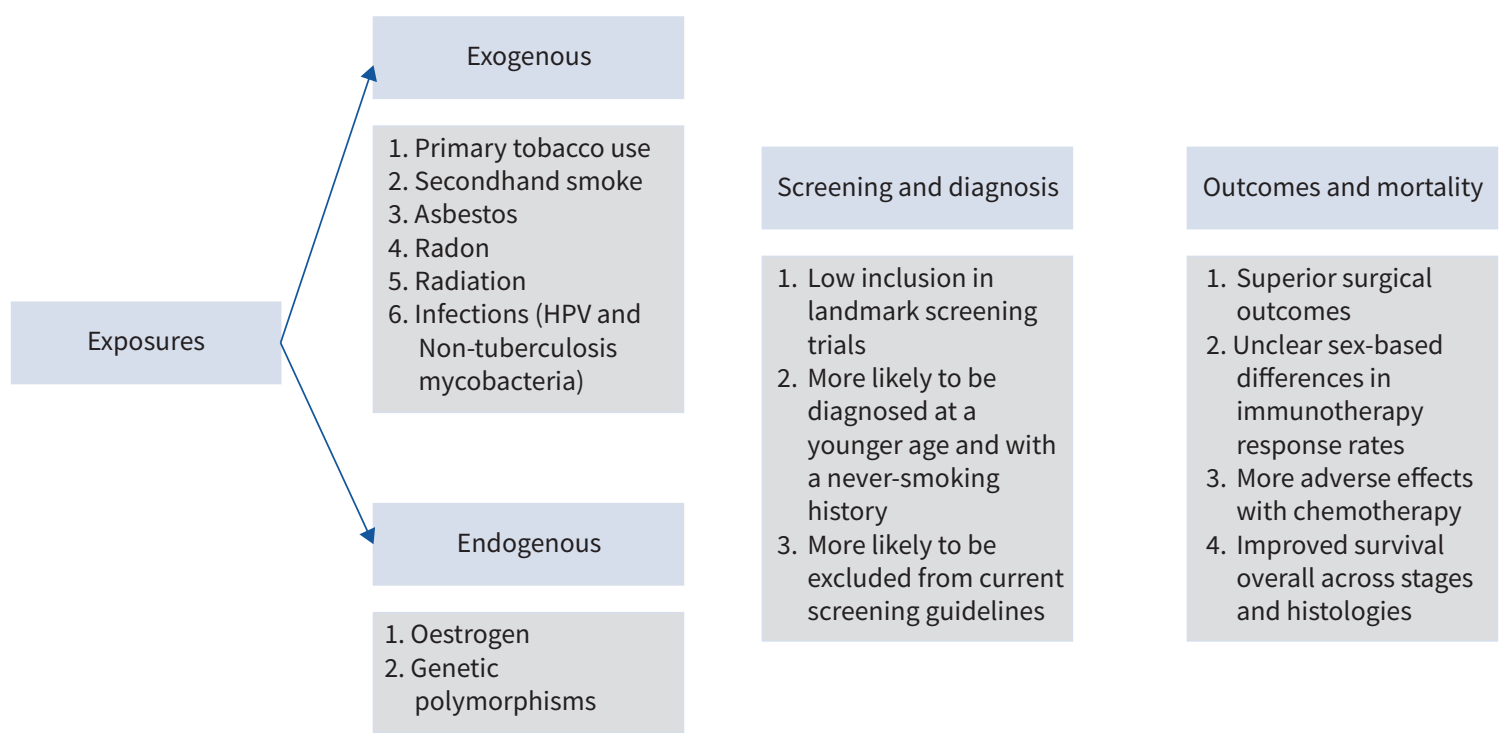

FIGURE 1 Summary of the unique exogenous and endogenous risk factors, screening considerations and outcome differences women with lung cancer face in comparison to men. HPV: human papillomavirus.

Risk factors and epidemiology

Smoking patterns: a delayed rise and an unequal fall

While there has been a notable rise in the incidence of lung cancer in never-smokers, the predominant risk factor for the development of lung cancer worldwide remains tobacco use. Cigarette smoking contributes to about $80 \%$ of annual lung cancer deaths in the United States and the majority of men and women diagnosed with lung cancer globally have a history of tobacco use [1]. In many parts of the world, tobacco use has declined as a result of expansive public health campaigns and awareness regarding the link of tobacco to lung cancer. In the US, in tandem with declining smoking rates, incidence of lung cancer has declined from 70.6 to 55.2 cases per 100000 people over the last two decades [9]. However, the rate of decline for both smoking rates and lung cancer incidence occurred later and has been slower for women compared to men [10]. In the European Union, smoking rates are declining in both sexes [11], but again the pace has been slower for women. In contrast, in many developing nations, smoking rates remain alarmingly high, leading to rising rates of lung cancer incidence, particularly in women [12]. Continued widespread public health awareness campaigns, such as targeted messaging for women, are essential to help curb the rise of tobacco use [3].

Among smokers, men may have a slightly higher increased risk of developing lung cancer than women $[12,13]$, although it is unclear if the pathogenesis of lung cancer in women who smoke compared to men who smoke is a distinct process. A number of studies have evaluated whether women may be more susceptible to the carcinogens in tobacco smoke. Specifically, prospective cohort studies and multiple meta-analyses have concluded that women do not appear more susceptible than men when adjusting for tobacco exposure [3, 4]. Prescott et al. [14] evaluated the risk of lung cancer associated with smoking status and quantification of tobacco use and found that lung cancer incidence rates were higher in men compared to women in those who had ever smoked. BAIN et al. [15] evaluated patients with a new diagnosis of lung cancer and found no differences between men and women regarding rates of lung cancer incidence once adjusted for smoking patterns. In contrast, however, some studies have shown that there may be some sex-based genomic differences in patients who smoke and develop lung cancer. For example, among women with lung cancer, those with a history of tobacco use have been found to more frequently harbour a tp53 point mutation when compared to those who have never smoked, and this finding does not appear to be consistent in men [2, 16]. Mutations in the glutathione s-transferase Mu 1 gene are also more common in women and have been linked to the development of lung cancer in smokers [17]. Limitations to these studies, however, include uncertain characterisation of tobacco exposure, as certain elements such as depth of inhalation and tar content of cigarettes are not readily or easily measured [15].

\section{Exogenous exposures}

Secondhand smoke

Secondhand smoke may be the third most common known risk factor for the development of lung cancer after primary tobacco use and radon. Current studies evaluating this link in women are mixed. One pooled 
analysis of 37 studies that aimed to evaluate the impact of secondhand smoke on lung cancer found that women whose spouses smoked had a $24 \%$ increased risk of developing lung cancer [18]. A secondary cohort analyses analysed data collected from the UK Million Women Study and evaluated the link between various risk factors and the development of lung cancer among women. The analysis did not identify secondhand smoke as a significant risk factor in lung cancer development among women who had never smoked; however, extent and duration of exposure was not characterised [5]. Quantifying exposure to secondhand smoke at a population level is even more challenging than quantifying tobacco use; thus, major conclusions are difficult to draw even in well-designed prospective or retrospective cohort analyses.

\section{Radon}

The Environmental Protection Agency estimates that radon is the second most common known exposure to cause lung cancer after tobacco, and considered to be the number one cause among non-smokers. Radon is a known radioactive compound present in natural soil, rock and water [19]. Occupational exposures to radon through mining have been associated with the development of lung cancer in prospective cohort studies. Extrapolation of this data to the residential setting is now well described in a number of casecontrol studies. One such study conducted in the state of New Jersey in the 1980s specifically evaluated the association between radon exposure and development of lung cancer in women. While the study did not demonstrate a significant difference in lung cancer development between women with a radon exposure and women without an exposure, it did reveal a trend of increasing risk of lung cancer with increasing exposure levels [20]. A pooled analysis incorporating seven case-control studies similarly demonstrated that there was a linear trend in the odds of developing lung cancer with higher radon exposure in the residential setting [21].

\section{Asbestos}

Asbestos is a common occupational and environmental hazard and is a known carcinogen. It has a well-known and nearly universal association with the development of mesothelioma, but is also commonly associated with the development of lung cancer. Asbestos was widely used in building, textiles and insulation materials until the late 1900s, and exposures have been described both in occupational and non-occupational settings, with often a long latency period (30 years or more) between exposure and development of cancer [22]. While the occupational risk of lung cancer from asbestos has been described with higher frequency in men, one meta-analysis found that women were at slightly higher risk compared to men of developing lung cancer from a non-occupational exposure to asbestos [23]. There is a paucity of cohort studies evaluating the risk of non-occupational asbestos exposure with the development of lung cancer; thus the severity of this risk, particularly in women, may be underrepresented currently.

\section{Radiation}

Prior radiation exposure is a well-described iatrogenic risk factor for the development of second primary lung cancer. Hodgkin's lymphoma and breast cancer have both been associated with the development of second primary lung cancer in patients who underwent radiation therapy [24-26]. Breast cancer in particular is also experiencing a demographic shift with rising incidence rates in younger patients, with increasing concern regarding the long-term side effects of early radiation exposure.

\section{Coal}

Indoor cooking fumes are another possible risk factor based on gender roles, where it is predominantly women who are exposed as compared to men. One study in Asia demonstrated that women who cook with coal have five times increased odds of developing lung cancer compared to women who do not [27]. Aside from coal, indoor cooking oils lead to the formation of polycyclic aromatic hydrocarbons (PAHs), which are known carcinogens. Women in developing areas of the world, particularly where indoor ventilation may be minimal, may be at risk for cumulative exposure to PAHs [3, 17, 28].

\section{Infection}

Likely risk factors for the pathogenesis of lung cancer may also include infections. The two most common infections studied are human papillomavirus (HPV) and mycobacterial infections (both tuberculosis and non-tuberculosis mycobacterium) [28-30]. These infections, which are more common on the Asian subcontinent, are potentially modifiable risk factors. One study in Taiwan demonstrated that among non-smokers, women were more likely to have HPV-positive lung cancer than men, and more likely to have adenocarcinoma compared to women who were not HPV-positive [31]. There are three additional published case studies specifically evaluating the impact of HPV 16/18 infection on lung cancer in never-smoking women, all of which have demonstrated that women with a history of HPV have increased odds for lung cancer development [32]. 
Tuberculosis (TB) has also been proposed as an independent risk factor for lung cancer development. Patients with a history of TB had a 1.7-fold increased relative risk of developing lung cancer compared to patients without TB history in one meta-analysis, although it is not clear that this risk is higher in women [33]. This relationship has also been demonstrated in non-tuberculosis mycobacteria, which has been shown to have some prevalence among patients diagnosed with lung cancer, particularly among women [34]. There are no large prospective studies that have replicated these findings, but the risk of chronic scarring to the lung from underlying granulomatous disease may be an important and potentially modifiable risk factor for lung cancer, particularly in the developing world [29].

\section{Endogenous exposures}

Genetic mutations

There are some well-described genetic variations between the two sexes in the non-smoking population. Polymorphisms in the cytochrome P450, family 1, subfamily A, polypeptide 1 gene which lead to defects in DNA repair, overexpression of X-linked gastrin releasing peptide receptor and mutations in p53 have all been associated with increased pathogenesis of lung cancer in women. Women are also at increased risk compared to men of developing lung cancer with a driver mutation, particularly epidermal growth factor receptor (EGFR), anaplastic lymphoma kinase (ALK) and Kirsten rat sarcoma mutations (KRAS) [3, 16, 17, 29]. Family history of lung cancer has clearly been demonstrated to be a significant risk factor in the development of lung cancer, but it is unclear if that effect is more pronounced in women [5, 35].

\section{Oestrogen}

The impact of oestrogen on lung cancer has been a topic of ongoing study. One retrospective study utilising the Surveillance, Epidemiology, and End Results data demonstrated that premenopausal women diagnosed with lung cancer tended to present with more extensive disease and were more likely to develop adenocarcinoma compared to postmenopausal women [36]. The oestrogen receptor is known to be overexpressed in many lung cancers, but there is no clear consensus on the impact of oestrogen and hormone replacement therapy on the development of lung cancer in women [5, 17, 28]. GREISER et al. [37] analysed the associations between ever-use of hormonal therapy and risk of lung cancer in a meta-analysis; ever-use was associated with a $27 \%$ decrease in lung cancer risk irrespective of smoking history. However, in a subgroup analysis of patients who developed adenocarcinoma, there was an increased association with ever-use of HRT. Larger, prospective studies on the role of both endogenous and exogenous oestrogen are needed to help more definitively characterise the link between hormones and lung cancer development.

\section{Screening and diagnosis}

Many randomised controlled trials evaluated the impact of screening for lung cancer with low-density computed tomography (LDCT) on mortality. The largest prospective study was the National Lung Screening Study (NLST) conducted in the United States with 55000 participants. Women comprised $40 \%$ of those enrolled on the trial. The results of the NLST led to the publication of the United States Preventative Services Task Force (USPSTF) category B recommendation in 2013 that all men and women between the ages of 55-80 with at least a 30 pack-year smoking history undergo annual screening for lung cancer with a low-dose computed tomography (CT) scan [38]. The second largest trial that evaluated the impact of LDCT on outcomes was the NELSON study, which also enrolled patients with a significant smoking history and was conducted in the Netherlands and Belgium [39]. Women comprised only 16\% of trial enrollees and were not included in the main outcome analysis. Canada and the European Union have adopted similar guidelines based on the NLST and NELSON data and their own smaller randomised controlled trials that effectively mirror the NLST study in enrolling high-risk patients with a significant smoking history. Even in Asia, where there is a higher percentage of never-smokers who develop lung cancer, there are a few expert consensus guidelines that largely mirror the recommendations borne out of the NLST and apply only to patients with a significant smoking history [40]. There are no worldwide consensus guidelines on lung cancer screening, nor are there any nationally sponsored programmes to promote lung cancer screening. Of note, no current guidelines incorporate additional risk factors for lung cancer besides smoking history, and there are no sex-specific differences in guidelines to date.

Between 2010 and 2015, the National Cancer Institute reported that only 5.9\% of all patients eligible per the USPSTF guidelines underwent an LDCT [41], although there is wide variation by state [42, 43]. Women were slightly more likely to undergo screening than men (6.3 versus 5.6\%), but on the contrary, women eligible for lung cancer screening are also less likely to discuss LDCT with their providers [44], despite consistently being more likely to confer a mortality benefit from these screening modalities. In the German LUSI trial, which enrolled 4052 participants, women who underwent screening with an LDCT had a statistically significant reduction in mortality (hazard ratio (HR) 0.31, p=0.04), whereas men did not (HR 0.94, p=0.81) [45]. A similar effect was demonstrated in a subgroup analysis of the NLST, where 
women screened by CT had lower lung cancer related risk of mortality (HR 0.73) compared to men (HR 0.92, $\mathrm{p}=0.08$ ) [38]. In the NELSON trial, women who underwent interval LDCT screening had a non-statistically significant reduction in lung cancer related mortality at 10 years (HR 0.67), whereas there were no differences in lung cancer related mortality among men who underwent regular CT screening (HR 1.01), although the trial was not powered to detect differences in mortality [39]. The radiographic features and operability of identified nodules may vary between men and women. In a retrospective analysis of the NLST data, women with ground glass nodules had a higher risk of developing lung cancer compared to men [46]. Given that women are more likely to be diagnosed with adenocarcinoma, which is typically slow growing, it is possible that an optimal screening interval in women may be more frequently than annually, although there is currently insufficient evidence to support this.

The lack of guidelines and risk assessments for light or never smokers predisposes women with lung cancer in particular to be missed with current screening recommendations. Between 50 and $80 \%$ of women diagnosed with lung cancer have been demonstrated to not meet the screening criteria outlined by the USPSTF and other national agencies, a consistent metric worldwide given the high rates of light or never smokers in women diagnosed with lung cancer [7, 47, 48]. In addition, on average, women are diagnosed at younger ages than men, and the incidence of lung cancer in younger patients across sexes is rising [1, 2]. As current guidelines do not adequately identify particular high-risk groups, specifically women and under-represented minorities, the USPSTF published modified guidelines in 2021, liberalising the pack year history to 20 and the lower age limit to 50 years [6]. While the percentage of both men and women eligible for screening will increase with expanded eligibility criteria [49], a rising proportion of the population will continue to be excluded from the screening criteria given the rapidly and constantly changing demographics and epidemiology of lung cancer worldwide. In particular, young women who are still of childbearing age are at increasing risk of being left out of screening guidelines; however, reassuringly, the development of lung cancer during pregnancy is quite rare [50].

Younger patients who are screened for lung cancer could stand to benefit from years of additional life with earlier detection and cure [6]. However, in this population in particular, cumulative radiation exposure also contributes to a lifetime risk of developing lung cancer. One study estimated that this risk in women after three LDCTs amounts to about 1.5 cases of lung cancer alone per 1000 exposed [51]. In addition to the risk of ongoing radiation exposure, the benefits of lung cancer screening must be weighed against the risks of detecting a false positive, which can lead to unnecessary invasive interventions, psychological stress and an increased cost burden to the system.

\section{Outcomes}

Lung cancer has now surpassed breast cancer as the number one cancer-related cause of death worldwide in both women and men [11, 35]. In the United States, although mortality from lung cancer has declined steadily over the last two decades due to earlier detection and significant treatment advances such as targeted agents and immunotherapy [3], the mortality of lung cancer in women has still increased five-fold overall since 1950 [52] and is declining at a slower rate than it is in men [29]. In some areas of the world, such as Brazil, mortality from lung cancer is still on the rise in women whereas it is declining in men [11]. Despite these variations in mortality trends, women have been shown consistently over time to have overall improved survival rates compared to men when tobacco exposure, stage at diagnosis, age and treatment modality are accounted for [12, 17, 53-58].

\section{Early stage}

Women are likely to be diagnosed with lung cancer at an earlier stage than men [8, 59]. Sex-based differences in outcomes of surgical resection have also been widely reported. Tong et al. [54] demonstrated that in a large national database of thoracic surgery patients who underwent surgical resection for lung cancer between 2002 and 2010, women had a lower 30-day mortality (1.5 versus 3.0\%, $\mathrm{p}<0.001$ ) and a statistically significantly lower rate of nearly all recorded post-operative complications; however, the women were younger and generally had superior baseline health compared to their male counterparts. In a retrospective study in Finland, women who had a resection for early-stage lung cancer experienced superior survival rates at 5, 10 and 14 years compared to men, in addition to lower morbidity, 30-day mortality and complications rates [60]. Similar results were shown in a retrospective study in Norway, where male sex was a risk factor that conferred a $27 \%$ increase in overall mortality following surgical resection for early-stage lung cancer [61].

Stereotactic body radiation therapy (SBRT) is another potentially curative option for early-stage lung cancer that is deemed to not be operable either because of the anatomic location of the tumour or, more commonly, because the patient's underlying comorbidities confer an excessive risk of morbidity and 
mortality with surgery. While not extensively studied, there appear to be no major differences in receipt of SBRT versus surgery between men and women with early-stage non-small cell lung cancer (NSCLC) [62, 63]. Sex-specific survival data for patients receiving definitive SBRT with curative intent is limited. The largest retrospective study evaluated outcomes in patients over the age of 70 undergoing SBRT for early-stage lung cancer compared to patients who were observed without treatment. Men treated with SBRT did not have an improved survival benefit compared to those who underwent observation, but women treated with SBRT did have slightly improved survival rates compared to women who underwent observation [64].

\section{Advanced stage}

Similarly, women with advanced stage cancer have improved survival rates compared to men. This survival benefit persists when histology, stage at diagnosis, smoking history and age are taken into account [8, 55]. Wheatley-Price et al. [65] conducted a pooled analysis in 2010 of five randomised trials of platinum-based chemotherapy in advanced NSCLC and found that women had a higher response rate (42\% versus $40 \%$ ) and a longer overall survival (OS) (9.6 versus 8.6 months). More recently, there has been interested in identifying sex-based differences in response rates to immunotherapy in NSCLC, and results of such studies have been mixed. In one pooled analysis of five of the landmark immunotherapy trials in NSCLC, monotherapy with anti-PD1 inhibitors significantly improved progression-free survival (PFS) when compared with chemotherapy in men enrolled on the trials, but there was no PFS difference between the two groups found in women [66]. However, in another pooled analysis of eight trials in NSCLC evaluating the impact of chemotherapy plus immunotherapy versus chemotherapy alone, women had a slight survival advantage when receiving combined chemotherapy and immunotherapy compared to men [67]. There are also sex-based differences in the development of side effects of systemic therapy. Women have been demonstrated to be more likely to experience side effects from chemotherapy, and there are evolving data to suggest that women receiving immunotherapy may be at higher risk of immune-related adverse events [68, 69]. Duma et al. [70] found in a retrospective study of patients diagnosed with NSCLC and melanoma that women were more likely to develop immune-related adverse events compared to men and, interestingly, that this risk was higher in premenopausal women. Sex-based differences in response to immunotherapy may also be explained by biological differences in the tumour immune microenvironment. For example, there has been increasing evidence that the gut microbiome, which may differ significantly between men and women based on both environmental and endogenous factors, plays a role in immunotherapy response rates [71]. The higher predisposition of women to develop autoimmune conditions has also been linked to differences in the gut microbiome [72]; it is not yet clear whether sex-based differences in the microbiome directly translate to differences in immunotherapy response rates.

In more recent landmark studies, there have been no significant differences in outcomes between women and men. Women have been shown to have higher response rates to first-generation EGFR inhibitors [66]. In the landmark FLAURA trial, which demonstrated superior PFS and OS of osimertinib compared to first-generation EGFR tyrosine kinase inhibitors, women tended towards having improved survival (HR for progression 0.40 versus 0.58 in men), but this difference was not statistically significant [73]. Similarly, in the landmark study showing the superiority of alectinib over crizotinib in overall survival for patients harbouring an ALK mutation, women tended towards having improved survival compared to men, but this difference was not statistically significant (HR for disease progression 0.39 versus 0.61 in men) [74].

Small-cell lung cancer (SCLC) is less extensively studied, particularly as it is uncommon in women, but there are some limited data to suggest that women have improved outcomes at both limited and extensive stages compared to men. In one pooled analysis of 10 trials conducted through the Southwestern Oncology Group of 2580 patients with SCLC, among patients with limited stage disease, women had improved survival compared to men (24.4 months versus 17.7 months). However, this same analysis did not find sex-based differences in survival among women with extensive stage disease and was conducted over two decades ago [75]. A more recent retrospective study conducted by Duma et al. found that women have an improved median OS in both limited stage SCLC (15.2 versus 12.7 months) and extensive stage SCLC (6.4 versus 5.7 months), although the latter benefit was modest [76].

\section{Conclusions}

The pathogenesis, screening, diagnosis, outcomes and treatment side effects for women diagnosed with lung cancer have unique and distinct features compared to men but are currently not fully understood. These sex-based differences suggest that lung cancer may be increasingly considered a distinct disease in women. These differences are increasingly important for cancer researchers and clinicians to understand, as the incidence of lung cancer continues to rise globally in younger, never-smoker patients, a demographic group that far favours women compared to men. Current screening guidelines, which unfortunately have 
had similarly low uptake in men and women in the United States and have not been adopted on a global scale, reflect the epidemiologic patterns of lung cancer from nearly half a century ago and are expected to miss more than half of all women diagnosed with lung cancer worldwide. While women have improved mortality compared to men across lung cancer types and stages, the changing demographic patterns of lung cancer globally threaten to shift this paradigm over the coming decades. Population-level studies on the unique exposures faced by women, expansion of screening guidelines to incorporate risks other than tobacco, and ongoing emphasis of enrolment of women and reporting of sex-based differences in pivotal clinical trials will help address existing sex-based disparities in lung cancer.

Provenance: Commissioned article, peer reviewed.

Previous articles in this series: No. 1: Cheron C, McBride SA, Antigny F, et al. Sex and gender in pulmonary arterial hypertension. Eur Respir Rev 2021; 30: 200330. No. 2: LoMauro A, Aliverti A. Sex and gender in respiratory physiology. Eur Respir Rev 2021; 30: 210038. No 3: Chowdhury NU, Guntur VP, Newcomb DC, et al. Sex and gender in asthma. Eur Respir Rev 2021; 30: 210067. No 4: Kawano-Dourado L, Glassberg MK, Assayag D, et al. Sex and gender in interstitial lung diseases. Eur Respir Rev 2021; 30: 210105. No 5: Dominelli PB, Molgat-Seon Y. Sex, gender and the pulmonary physiology of exercise. Eur Respir Rev 2022; 31: 210074.

Conflict of interest: M. Ragavan has nothing to disclose. M.I. Patel reports support for the present manuscript from the $\mathrm{NIH}$ in part for time dedicated to the manuscript. Outside the submitted work: grants or contracts received from NIHMD K23MD013474.

\section{References}

1 Jemal A, Miller KD, Ma J, et al. Higher lung cancer incidence in young women than young men in the United States. N Engl J Med 2018; 378: 1999-2009.

2 Fidler-Benaoudia MM, Torre LA, Bray F, et al. Lung cancer incidence in young women vs. young men: A systematic analysis in 40 countries. Int J Cancer 2020; 147: 811-819.

3 Kligerman S, White C. Epidemiology of lung cancer in women: risk factors, survival, and screening. AJR Am J Roentgenol 2011; 196: 287-295.

$4 \quad$ Barrera-Rodriguez R, Morales-Fuentes J. Lung cancer in women. Lung Cancer Targets Ther 2012; 3: 79-89.

5 Pirie K, Peto R, Green J, et al. Lung cancer in never smokers in the UK Million Women Study. Int J Cancer 2016; 139: 347-354.

6 Colson YL, Shepard J-AO, Lennes IT. New USPSTF guidelines for lung cancer screening: better but not enough. JAMA Surg 2021; 156: 513-514.

7 Velez Martinez C, Thurlapati A, Hirani S, et al. Assessing risk-based LDCT screening strategies versus the current USPSTF lung cancer screening recommendations at an institutional setting. J Clin Oncol 2020; 38: e13572.

8 Sagerup CMT, Småstuen M, Johannesen TB, et al. Sex-specific trends in lung cancer incidence and survival: a population study of 40118 cases. Thorax 2011; 66: 301-307.

9 US Cancer Statistics. Data visualizations https://gis.cdc.gov/Cancer/USCS/\#/AtAGlance/.

10 Houston KA, Henley SJ, Li J, et al. Patterns in lung cancer incidence rates and trends by histologic type in the United States, 2004-2009. Lung Cancer Amst Neth 2014; 86: 22-28.

11 Barta JA, Powell CA, Wisnivesky JP. Global epidemiology of lung cancer. Ann Glob Health 2019; 85: 8.

12 Thun MJ, Hannan LM, Adams-Campbell LL, et al. Lung cancer occurrence in never-smokers: an analysis of 13 cohorts and 22 cancer registry studies. PLoS Med 2008; 5: e185.

13 Marang-van de Mheen PJ, Smith GD, Hart CL, et al. Are women more sensitive to smoking than men? Findings from the Renfrew and Paisley study. Int J Epidemiol 2001; 30: 787-792.

14 Prescott E, Osler M, Hein HO, et al. Gender and smoking-related risk of lung cancer. The Copenhagen Center for Prospective Population Studies. Epidemiol Camb Mass 1998; 9: 79-83.

15 Bain C, Feskanich D, Speizer FE, et al. Lung cancer rates in men and women with comparable histories of smoking. J Natl Cancer Inst. 2004; 96: 826-834.

16 Yim S-H, Chung Y-J. Molecular epidemiology of female lung cancer. Cancers 2011; 3: 1861-1876.

17 Berardi R, Verdecchia L, Paolo MD, et al. Women and lung cancer: clinical and molecular profiling as a determinate for treatment decisions: a literature review. Crit Rev Oncol Hematol 2009; 69: 223-236.

18 Hackshaw AK, Law MR, Wald NJ. The accumulated evidence on lung cancer and environmental tobacco smoke. BMJ 1997; 315: 980-988.

19 US Environmental Protection Agency. Health Risk of Radon www.epa.gov/radon/health-risk-radon

20 Centers for Disease Control (CDC). Lung Cancer and Exposure to Radon in Women - New Jersey. MMWR 1989; 38: 715-718. 
21 Krewski D, Lubin JH, Zielinski JM, et al. A combined analysis of North American case-control studies of residential radon and lung cancer. J Toxicol Environ Health A 2006; 69: 533-597.

22 Kwak K, Kang D, Paek D. Environmental exposure to asbestos and the risk of lung cancer: a systematic review and meta-analysis. Occup Environ Med 2021; in press [https://doi.org/10.1136/oemed-2020-107222].

23 Cheng YY, Rath EM, Linton A, et al. The current understanding of asbestos-induced epigenetic changes associated with lung cancer. Lung Cancer Targets Ther 2020; 11: 1-11.

24 Tucker MA, Coleman CN, Cox RS, et al. Risk of second cancers after treatment for Hodgkin's disease. $N$ Engl J Med 1988; 318: 76-81.

25 Kaufman EL, Jacobson JS, Hershman DL, et al. Effect of breast cancer radiotherapy and cigarette smoking on risk of second primary lung cancer. J Clin Oncol 2008; 26: 392-398.

26 Wang R, Yin Z, Liu L, et al. Second primary lung cancer after breast cancer: a population-based study of 6,269 women. Front Oncol 2018; 8: 427.

27 Hosgood HD, Boffetta P, Greenland S, et al. In-home coal and wood use and lung cancer risk: a pooled analysis of the International Lung Cancer Consortium. Environ Health Perspect 2010; 118: 1743-1747.

28 North CM, Christiani DC. Women and lung cancer: what's new? Semin Thorac Cardiovasc Surg 2013; 25: 87-94.

29 MacRosty CR, Rivera MP. Lung cancer in women: a modern epidemic. Clin Chest Med 2020; 41: 53-65.

30 de Groot PM, Wu CC, Carter BW, et al. The epidemiology of lung cancer. Transl Lung Cancer Res 2018; 7: 220-233.

31 Cheng YW, Chiou HL, Sheu GT, et al. The association of human papillomavirus 16/18 infection with lung cancer among nonsmoking Taiwanese women Cancer Res 2001; 61: 2799-2803.

32 Bae J-M, Kim EH. Human papillomavirus infection and risk of lung cancer in never-smokers and women: an 'adaptive' meta-analysis. Epidemiol Health 2015; 37: e2015052.

33 Brenner DR, McLaughlin JR, Hung RJ. Previous lung diseases and lung cancer risk: a systematic review and meta-analysis. PLOS ONE 2011; 6: e17479.

34 Tamura A, Hebisawa A, Kusaka K, et al. Relationship between lung cancer and Mycobacterium avium complex isolated using bronchoscopy. Open Respir Med J 2016; 10: 20-28.

35 Isla D, Majem M, Viñolas N, et al. A consensus statement on the gender perspective in lung cancer. Clin Transl Oncol Off Publ Fed Span Oncol Soc Natl Cancer Inst Mex 2017; 19: 527-535.

36 Moore KA, Mery CM, Jaklitsch MT. Menopausal effects on presentation, treatment, and survival of women with non-small cell lung cancer. Ann Thorac Surg 2003; 76: 1789-1795.

37 Greiser CM, Greiser EM, Dören M. Menopausal hormone therapy and risk of lung cancer-Systematic review and meta-analysis. Maturitas 2010; 65: 198-204.

38 Pinsky PF, Church TR, Izmirlian G, et al. The national lung screening trial: results stratified by demographics, smoking history and lung cancer histology. Cancer 2013; 119: 3976-3983.

39 de Koning HJ, van der Aalst CM, de Jong PA, et al. Reduced lung-cancer mortality with volume CT screening in a randomized trial. N Engl J Med 2020; 382: 503-513.

40 Zhou Q, Yaguang F, Wang Y et al. China national lung cancer screening guideline with low-dose computed tomography (2018 version). Zhongguo Fei Ai Za Zhi Chin J Lung Cancer 2018; 21: 67-75.

41 Han SS, Chow E, ten Haaf K, et al. Disparities of national lung cancer screening guidelines in the US population. JNCI J Natl Cancer Inst 2020; 112: 1136-1142.

42 Fedewa SA, Kazerooni EA, Studts JL, et al. State variation in low-dose computed tomography scanning for lung cancer screening in the United States. JNCI J Natl Cancer Inst 2021; 113: 1044-1052.

43 Siegel DA, Fedewa SA, Henley SJ, et al. Proportion of never smokers among men and women with lung cancer in 7 US States. JAMA Oncol 2021; 7: 302-304.

44 Warner ET, Lathan CS. Race and sex differences in patient provider communication and awareness of lung cancer screening in the health information National Trends Survey, 2013-2017. Prev Med 2019; 124: 84-90.

45 Becker N, Motsch E, Trotter A, et al. Lung cancer mortality reduction by LDCT screening-Results from the randomized German LUSI trial. Int J Cancer 2020; 146: 1503-1513.

46 Duan F, Chysanthopoulou S, De Mello S. Lung nodule consistency and relative risk of future lung cancer diagnosis: does sex matter? Radiological Society of North America Annual Meeting, Abstract SSA 0402.

47 Vu C, Lin S, Chang C-F. Gender gaps in care: lung cancer screening criteria in women. Chest 2019; 156 : A407.

$48 \mathrm{Ji} \mathrm{G}$, Bao T, Li Z, et al. Current lung cancer screening guidelines may miss high-risk population: a real-world study. BMC Cancer 2021; 21: 50.

49 Reese TJ, Schlechter CR, Potter LN, et al. Evaluation of revised US preventive services task force lung cancer screening guideline among women and racial/ethnic minority populations. JAMA Netw Open 2021; 4: e2033769.

50 Sarıman N, Levent E, Yener NA, et al. Lung cancer and pregnancy. Lung Cancer 2013; 79: 321-323.

51 Brenner DJ. Radiation risks potentially associated with low-dose CT screening of adult smokers for lung cancer. Radiology 2004; 231: 440-445.

52 Zang EA, Wynder EL. Differences in lung cancer risk between men and women: examination of the evidence. J Natl Cancer Inst 1996; 88: 183-192. 
53 International Early Lung Cancer Action Program Investigators, Henschke Cl, Yip R, et al. Women's susceptibility to tobacco carcinogens and survival after diagnosis of lung cancer. JAMA 2006; 296: 180-184.

54 Tong BC, Kosinski AS, Burfeind WR, et al. Sex differences in early outcomes after lung cancer resection: analysis of the Society of Thoracic Surgeons' general thoracic database. J Thorac Cardiovasc Surg 2014; 148: 13-18.

55 Visbal AL, Williams BA, Nichols FC, et al. Gender differences in non-small-cell lung cancer survival: an analysis of 4,618 patients diagnosed between 1997 and 2002. Ann Thorac Surg 2004; 78: 209-215.

56 Hellyer JA, Patel MI. Sex disparities in lung cancer incidence: validation of a long-observed trend. Transl Lung Cancer Res 2019; 8: 543-545.

57 Wisnivesky JP, Halm EA. Sex differences in lung cancer survival: do tumors behave differently in elderly women? J Clin Oncol 2007; 25: 1705-1712.

58 Elkbuli A, Byrne MM, Zhao W, et al. Gender disparities in lung cancer survival from an enriched Florida population-based cancer registry. Ann Med Surg 2020; 60: 680-685.

59 Sakurai H, Asamura H, Goya T, et al. Survival differences by gender for resected non-small cell lung cancer: a retrospective analysis of 12,509 cases in a Japanese lung cancer registry study. J Thorac Oncol 2010; 5: 1594-1601.

60 Lautamäki A, Gunn J, Sipilä J, et al. Women have a higher resection rate for lung cancer and improved survival after surgery. Interact Cardiovasc Thorac Surg 2021; 32: 889-895.

61 Strand T-E, Bartnes K, Rostad H. National trends in lung cancer surgery. Eur J Cardiothorac Surg 2012; 42: 355-358.

62 Dong B, Wang J, Xu Y, et al. Comparison of the efficacy of stereotactic body radiotherapy versus surgical treatment for early-stage non-small cell lung cancer after propensity score matching. Transl Oncol 2019; 12: 1032-1037.

63 Lin Q, Sun X, Zhou N, et al. Outcomes of stereotactic body radiotherapy versus lobectomy for stage I non-small cell lung cancer: a propensity score matching analysis. BMC Pulm Med 2019; 19: 98.

64 Nanda RH, Liu Y, Gillespie TW, et al. Stereotactic body radiation therapy versus no treatment for early stage non-small cell lung cancer in medically inoperable elderly patients: a National Cancer Data Base analysis. Cancer 2015; 121: 4222-4230.

65 Wheatley-Price P, Blackhall F, Lee S-M, et al. The influence of sex and histology on outcomes in non-small-cell lung cancer: a pooled analysis of five randomized trials. Ann Oncol Off J Eur Soc Med Oncol 2010; 21: 2023-2028.

66 Pinto JA, Vallejos CS, Raez LE, et al. Gender and outcomes in non-small cell lung cancer: an old prognostic variable comes back for targeted therapy and immunotherapy? ESMO Open 2018; 3: e000344.

67 Conforti F, Pala L, Bagnardi V, et al. Sex-based heterogeneity in response to lung cancer immunotherapy: a systematic review and meta-analysis. JNCI J Natl Cancer Inst 2019; 111: 772-781.

68 Colli LM, Morton LM, Chanock SJ. Sex-related effect on immunotherapy response: implications and opportunities. JNCI J Natl Cancer Inst 2019; 111: 749-750.

69 Wang C, Qiao W, Jiang Y, et al. Effect of sex on the efficacy of patients receiving immune checkpoint inhibitors in advanced non-small cell lung cancer. Cancer Med 2019; 8: 4023-4031.

70 Duma N, Abdel-Ghani A, Yadav S, et al. Sex differences in tolerability to anti-programmed cell death protein 1 therapy in patients with metastatic melanoma and non-small cell lung cancer: are we all equal? Oncologist 2019; 24: e1148-e1155.

71 Kroemer G, Zitvogel L. The breakthrough of the microbiota. Nat Rev Immunol 2018; 18: 87-88.

72 Gomez A, Luckey D, Taneja V. The gut microbiome in autoimmunity: sex matters. Clin Immunol 2015; 159: 154-162.

73 Soria J-C, Ohe Y, Vansteenkiste J, et al. Osimertinib in untreated EGFR-mutated advanced non-small-cell lung cancer. N Engl J Med 2018; 378: 113-125.

74 Peters S, Camidge DR, Shaw AT, et al. Alectinib versus crizotinib in untreated ALK-positive non-small-cell lung cancer. N Engl J Med 2017; 377: 829-838.

75 Albain KS, Crowley JJ, LeBlanc M, et al. Determinants of improved outcome in small-cell lung cancer: an analysis of the 2,580-patient Southwest Oncology Group data base. J Clin Oncol Off J Am Soc Clin Oncol 1990; 8: 1563-1574.

76 Duma N, Ho TP, Durani U, et al. Exploring sex differences in small cell lung cancer: Is this a hormonal issue? J Clin Oncol 2019; 37: e20077. 